\title{
Dreiwöchige Intensivtherapie verbessert die Kommunikationsfähigkeit
}

Fragestellung: Führt eine dreiwöchige intensive Logopädie/ Sprachtherapie bei Patienten mit chronischer Aphasie nach einem Schlaganfall zu einer Verbesserung der verbalen Kommunikationsfähigkeit im Vergleich zur Standardverordnungspraxis?

Hintergrund: Bei zirka $20 \%$ aller Schlaganfallpatienten besteht eine chronische Aphasie, die häufig mit erheblichen Einschränkungen der Alltagsaktivitäten sowie der Lebensqualität verbunden ist und hohe Kosten verursacht. Mehrere Metaanalysen haben bereits Hinweise auf eine Wirksamkeit von mindestens fünf bis zehn Stunden Sprachtherapie pro Woche bei Aphasiepatienten erbracht, wobei die Aussagekraft der einzelnen Studien methodisch zum Beispiel durch kleine Fallzahlen oder eine geringe Therapieintensität eingeschränkt war $[1,2]$.

Breitenstein C, Grewe T, Flöel A et al. Intensive speech and language therapy in patients with chronic aphasia after stroke: a randomised, open-label, blinded-endpoint, controlled trial in a health-care setting. Lancet 2017; 389: 1528 - 38
Patienten und Methodik: In der prospektiven, multizentrischen Studie wurden 158 Schlaganfallpatienten (18 bis 70 Jahre; ischämischer Hirninfarkt, ICB oder SAB) mit chronischer Aphasie ( $\geq$ sechs Monate, tatsächliche Latenz im Median zirka zweieinhalb
Jahre) aus 19 deutschen Rehabilitationseinrichtungen (ambulant oder stationär) in einem Parallelgruppendesign 1:1 in zwei Gruppen randomisiert. Die Interventionsgruppe erhielt für mindestens drei Wochen intensive Sprachtherapie mit mindestens zehn Stunden pro Woche. Die Kontrollgruppe bekam erst mit einer Latenz von drei Wochen die gleiche Therapie. Das Gesamt-Follow-up betrug sechs Monate. Primärer Studienendpunkt war die Verbesserung der verbalen Kommunikationsfähigkeit am Ende der dreiwöchigen Intensivtherapie, gemessen mit einem standardisierten Testverfahren, im Vergleich zur Kontrollgruppe. Die Beurteilung des primären Endpunktes fand mittels Tonbandaufnahmen verblindet statt.

Ergebnisse: Verbale Kommunikationsfähigkeit und Lebensqualität verbesserten sich in der Intensivtherapiegruppe innerhalb von drei Wochen signifikant im Vergleich zur Kontrollgruppe. Die erreichten Effekte blieben auch über einen Zeitraum von sechs Monaten stabil. Während der Intensivtherapiephase gab es keine Studienabbrecher.

Schlussfolgerungen: Eine dreiwöchige intensive Sprachtherapie mit mindestens zehn Stunden pro Woche verbessert bei $<70$-jährigen Schlaganfallpatienten mit chronischer Aphasie die verbale Kommunikationsfähigkeit und ist der normalen Verordnungspraxis überlegen.

\section{- Kommentar von Andreas Bender, Burgau und München}

\section{Erhebliches Potenzial auch bei chronischer Aphasie}

Die FCET2EC-Studie ist eine sehr wichtige und methodisch sehr sorgfältig durchgeführte Studie auf dem Gebiet der neurologischen Rehabilitation. Sie bestätigt erstmals im Kontext einer realitätsnahen, multizentrischen klinischen Studie mit breiten Einschlusskriterien und patientenrelevanten klinischen Endpunkten die Ergebnisse bisheriger Metaanalysen, dass eine möglichst intensive Sprachtherapie wirksam und gegenüber der Versorgungsrealität überlegen ist. Auch wenn bei einer insgesamt mittleren bis hohen Effektstärke die durchschnittliche Verbesserung im verwendeten Kommunikationstest nur zirka $9 \%$ betrug, so muss bedacht werden, dass dies mit einer nur dreiwöchigen Intensivtherapie mehrere Jahre nach einem Schlaganfall gelang. Dass sich parallel auch die Lebensqualität besserte, spricht für die Alltagsrelevanz der erreichten sprachlichen Verbesserung.

Auch wenn die Studie nicht alle offenen Fragen beantworten konnte (z. B. optimale Therapiedosis, Langzeittherapieplanung, gesundheitsökonomische Bilanz), so belegt sie doch sehr eindrucksvoll, dass es nach einem Schlaganfall keine starren zeitlichen Rehabilitationszeitfenster gibt. Angesichts der großen Bedeutung der Sprache für die Lebensqualität bleibt zu hoffen, dass vergleichbare Therapiekonzepte in der Verordnungspraxis bewilligt, umgesetzt und weiter wissenschaftlich evaluiert werden.
Referenzen:
1. Allen L et al. Top Stroke Rehabil 2012; 19:523-35
2. Brady MC et al. Cochrane Database Syst Rev 2016; 6: CD000425

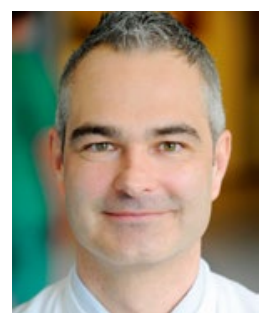

Prof. Dr. med. Andreas Bender, Burgau und München

Therapiezentrum Burgau und Neurologische Klinik, Klinikum der Universität München E-Mail: anbender@med.uni-muenchen.de 